\title{
Exome-wide association study identifies genetic polymorphisms of C12orf51, MYL2, and $A L D H 2$ associated with blood lead levels in the general Korean population
}

Sang-Yong Eom ${ }^{1}$, Myung Sil Hwang ${ }^{2}$, Ji-Ae Lim³ ${ }^{3}$ Byung-Sun Choi ${ }^{4}$,Ho-Jang Kwon ${ }^{3}$, Jung-Duck Park ${ }^{4}$, Yong-Dae Kim ${ }^{1 *}$ and Heon Kim ${ }^{1}$

\begin{abstract}
Background: Lead $(\mathrm{Pb})$ is a ubiquitous toxic metal present in the environment that poses adverse health effects to humans. Inter-individual variation in blood Pb levels is affected by various factors, including genetic makeup. However, limited data are available on the association between genetic variation and blood Pb levels. The purpose of this study was to identify the genetic markers associated with blood $\mathrm{Pb}$ levels in the Korean population.

Methods: The study subjects consisted of 1,483 healthy adults with no history of occupational exposure to $\mathrm{Pb}$. We measured blood $\mathrm{Pb}$ levels and calculated probable daily intake of $\mathrm{Pb}$ according to dietary data collected using 24-hour recall. We conducted exome-wide association screening using Illumina Human Exome-12v1.2 platform $(n=500)$ and a replication analysis using VeraCode Goldengate assay $(n=1,483)$.

Results: Among the 244,770 single nucleotide polymorphisms (SNPs) tested, 12 SNPs associated with blood Pb level were identified, with suggestive significance level $\left(P<1 \times 10^{-4}\right)$. In the Goldengate assay for replication, three SNPs (C12orf51 rs11066280, MYL2 rs12229654, and ALDH2 rs671) were associated with statistically suggestively significant differences in blood Pb levels. When stratified by drinking status, a potential association of C12orf51 rs11066280, MYL2 rs12229654, and ALDH2 rs671 with blood Pb level was observed only in drinkers. A marginally significant gene-environment interaction between ALDH2 rs671 and alcohol consumption was observed in relation to blood Pb levels. The effects of the three suggestively significant SNPs on blood Pb levels was dependent on daily calcium intake amounts.
\end{abstract}

Conclusions: This exome-wide association study indicated that C120rf51 rs11066280, MYL2 rs12229654, and ALDH2 rs671 polymorphisms are linked to blood Pb levels in the Korean population. Our results suggest that these three SNPs are involved in the determination of Pb levels in Koreans via the regulation of alcohol drinking behavior, and that their negative effects may be compensated by appropriate calcium intake.

Keywords: Single nucleotide polymorphism, Blood lead level, Exome-wide association study, Aldehyde dehydrogenase 2

\footnotetext{
* Correspondence: ydkim@chungbuk.ac.kr

${ }^{1}$ Department of Preventive Medicine, College of Medicine, Chungbuk

National University, 1 Chungdae-Ro, Seowon-Gu, Cheongju, Chungbuk

28644, Korea

Full list of author information is available at the end of the article
} 


\section{Background}

Lead $(\mathrm{Pb})$ is a global environmental health hazard that poses substantial risk to humans. Epidemiologic evidence indicates that increased $\mathrm{Pb}$ exposure is associated with hypertension [1], peripheral vascular disease [2], increased adult mortality [3], reproductive impairment [4], renal impairment [5], and altered immune function [6]. $\mathrm{Pb}$, a widely distributed metal, is a contaminant of ambient and workplace air, as well as of water, various foods, and tobacco $[7,8]$. Diet and air are major sources of $\mathrm{Pb}$ exposure for the general population [9]. Absorption of $\mathrm{Pb}$ occurs mainly via the respiratory and gastrointestinal tracts $[7,9]$. The rate of absorption of $\mathrm{Pb}$ via the gastrointestinal tract $(10-15 \%)$ is relatively lower than via inhalation (up to 50\%) [9]. Most $\mathrm{Pb}$ (about 90\%) absorbed by the body accumulates in the skeleton and is slowly released from the bones [7, 9]. The individual nutritional statuses of iron, calcium, and zinc are known to be important factors that affect the gastrointestinal absorption of $\mathrm{Pb}[10,11]$.

Inter-individual variation in blood $\mathrm{Pb}$ level is affected by various lifestyle and behavioral factors (i.e., smoking, drinking, dietary intake, and physical activity), as well as exposure dose $[7,9,12-14]$. In addition, genetic differences related to $\mathrm{Pb}$ toxicokinetics and toxicodynamics influence the body burden and toxic effects of this metal $[13,15]$. Several genes, such as aminolevulinate dehydratase $(A L A D)$ and vitamin D receptor $(V D R)$ [16-18], are involved directly or indirectly in Pb toxicokinetics; however, there are relatively few studies assessing the effects of genetic factors on blood Pb levels. Recently, the first genome-wide association study (GWAS) for blood $\mathrm{Pb}$ levels, which was conducted in cohorts from Australia and the UK, confirmed that genetic variation for the $A L A D$ gene plays a significant role in determining blood $\mathrm{Pb}$ levels [17]. However, to our knowledge, there is no study to identify a genetic marker for blood $\mathrm{Pb}$ levels in the Asian population, particularly in Korean individuals, using a genome-wide approach.

Therefore, the purpose of this study was to identify the genetic markers associated with blood $\mathrm{Pb}$ levels in the Korean population by exome-wide association screening and replication analysis.

\section{Methods}

\section{Study subjects}

The study subjects consisted of 1,483 healthy adults with no previous history of occupational exposure to $\mathrm{Pb}$. The subjects were selected from a cohort established by the Korean Research Project on the Integrated Exposure Assessment of Hazardous Substances for Food Safety (KRIEFS). The characteristics of this KRIEFS cohort and the method used to select the study subjects are described in detail in previous studies [19, 20]. Trained interviewers obtained demographic information as well as data on lifestyle factors such as smoking history, alcohol drinking habits, and food consumption, through a structured questionnaire. Venous blood was collected from subjects for genotypic analysis and evaluation of blood $\mathrm{Pb}$ levels. The collected blood was stored at $-80{ }^{\circ} \mathrm{C}$, as aliquots, until experimental use. This study was approved by the Institutional Review Board of Dankook University Hospital, Republic of Korea (IRB No. 2013-03-008), and informed consent was obtained from all participants.

\section{Estimation of $\mathrm{Pb}$ intake}

The probable daily intake of $\mathrm{Pb}$ was estimated from the dietary data collected using 24-hour recall. To determine the $\mathrm{Pb}$ content of the 135 food items, which represented the most frequently consumed food items based on Korean Health and Nutrition Examination Survey data, more than ten samples of each food item were gathered from seven metropolitan cities in South Korea. After pretreatment, the $\mathrm{Pb}$ content was measured using an inductively coupled plasma-mass spectrometer (ICP-MS, Perkin-Elmer, Elan 6100 DRC). The estimated amount of $\mathrm{Pb}$ intake for each food item was calculated by multiplying the food intake amount by its median content of $\mathrm{Pb}$, and the estimated daily total intake of $\mathrm{Pb}$ was calculated by adding the $\mathrm{Pb}$ intake amounts for all food items.

\section{Analysis of blood $\mathrm{Pb}$ level}

Blood $\mathrm{Pb}$ level was determined using a polarized Zeeman atomic absorption spectrophotometer (Model Z-2700, Hitachi, Tokyo, Japan). Briefly, blood was added to nitric acid and diluted with diammonium hydrogen phosphate and $1 \%$ Triton $\mathrm{X}-100$, followed by vigorous mixing. The detection limit was $0.059 \mu \mathrm{g} / \mathrm{dL}$ for blood $\mathrm{Pb}$. For samples with concentrations of blood $\mathrm{Pb}$ below the limit of detection, the concentration was substituted with the value for the limit of detection divided by the square root of 2 .

\section{Genotyping analysis \\ Exome-wide association screening}

Exome-wide association screening using an exome chip was performed to select SNPs associated with blood $\mathrm{Pb}$ levels in the Korean population. After randomly selecting 500 people from among the study subjects, exomewide association screening was conducted using a Human Exome chip v1.2 (Illumina, San Diego, USA), in which 244,770 SNP markers may be analyzed simultaneously. Human Exome chip is a commercial genotyping chip containing about 220,000 nonsynonymous SNPs that have putative functional exonic variants selected from whole-genome sequences. It also contains some SNPs located in the promoter region and splice site and 
also including disease-related tag markers such as various cancers, type 2 diabetes, and metabolic diseases recently identified in GWAS.

From quality control of human exome chip data, 783 SNPs were not found to be in Hardy-Weinberg equilibrium (HWE) $(P<0.001), 309$ SNPs had call rates less than $95 \%$, and 211,808 SNPs had extremely low minor allele frequencies of less than $1 \%$ (including monomorphic). The average call rate of all the samples was greater than $99.9 \%$, with a minimum value of $99.4 \%$. A blind replication test was conducted on 20 randomly selected samples; the error rate of all the samples was less than $0.05 \%$, and the average concordance rate was 99.96\%. For the 32,743 SNPs located on autosomal chromosomes with MAF of more than $1 \%$, sufficient call rates $(>95 \%)$, and in HWE $(P>0.001)$, the association with the markers of blood $\mathrm{Pb}$ levels was evaluated by multiple regression analysis using the program PLINK, and 12 suggestively significant SNPs $\left(P<1 \times 10^{-4}\right)$ were selected. Linkage disequilibrium (LD) block was determined using Haploview, and Tag SNPs were selected from the identified haplotype block. SNPs for which a probe could not be designed for the Goldengate assay were excluded from the final replication analysis. Finally, four SNPs (rs1268474, rs11066280, rs12229654, and rs671) on chromosomes 1 and 12 were selected by exome-wide association screening.

\section{Replication genotyping analysis}

Replication genotyping analysis was performed for the four selected SNPs using the VeraCode Goldengate assay (Illumina, San Diego, CA, USA) in all subjects. Analysis was performed on all 1,483 samples that passed DNA quality control (QC). The average call rate of the samples was 99.4\%, and that of the SNPs was 99.3\%. Two samples with call rates of less than $95 \%$ were excluded from the final analysis $(n=1,481)$. The results of the blind replication test on 19 randomly selected samples were highly reproducible, with an average concordance rate of $99.5 \%$.

\section{Statistical analysis}

The concentration of blood $\mathrm{Pb}$ was log-transformed for statistical analyses as this parameter was not normally distributed. Means of blood $\mathrm{Pb}$ levels for various genotypes were compared by analysis of variance. To test the effects of SNPs on blood Pb levels, univariate and multivariate regression models, with covariates such as age, sex, smoking status, drinking habits, and dietary $\mathrm{Pb}$ intake, were used. The multivariate regression model analysis was performed with two covariate combinations. One included age, gender, and smoking status as covariates, and the other additionally included drinking status and dietary intake of $\mathrm{Pb}$ as variables in the first model.
The correlation between dietary $\mathrm{Pb}$ intake and blood $\mathrm{Pb}$ levels was evaluated using the Spearman correlation coefficient. In addition, a stratification analysis was performed on drinking and calcium intake status to assess the effect of SNP on blood Pb level according to these two variables. The estimated amount of calcium intake was categorized into tertiles, and a stratified analysis was used to estimate the potential joint effects. $P$-values for interactions between the genotypes and dietary $\mathrm{Pb}$ or calcium intake were assessed, using the Wald test, for the cross-product term in a model containing the main effects of genotype and exposure variables. We used the Bonferroni correction for multiple tests ( $n=32,743$ tests) and set the statistical significance and suggestive threshold to $P$-values less than $1 \times 10^{-6}$ and $1 \times 10^{-4}$, respectively. Genetic association analyses were performed using PLINK v 1.07 software. A Manhattan plot of the exome-wide association study results was generated using Haploview 4.2 software. All other statistical analyses were performed using SPSS 23 (IBM, Armonk, NY, USA).

\section{Results}

The dietary $\mathrm{Pb}$ intake and blood $\mathrm{Pb}$ level were $20.43 \mu \mathrm{g} /$ day and $2.21 \mu \mathrm{g} / \mathrm{dL}$, respectively, and significantly higher in males than in females. The levels of both parameters were highest in the group aged 50-59; blood $\mathrm{Pb}$ levels were found to increase significantly with age. The dietary $\mathrm{Pb}$ intake and blood $\mathrm{Pb}$ levels were higher in smokers than in non-smokers, and in drinkers than in non-drinkers (Table 1).

To identify SNP markers associated with blood $\mathrm{Pb}$ levels, we performed an exome-wide association study of 244,770 SNPs via human exome chip analysis. The Manhattan plot, which was derived from the association analysis between blood $\mathrm{Pb}$ levels and SNPs using linear regression analysis with an additive genetic model, is shown in Fig. 1. The list of top 100 most significant SNPs associated with blood $\mathrm{Pb}$ level, as identified by exome-wide association screening, is provided in Additional file 1: Table S1. No SNP reached an exomewide significance level of association $\left(P<1 \times 10^{-6}\right)$. SNPs associated with blood $\mathrm{Pb}$ levels, with suggestively significant level $\left(P<1 \times 10^{-4}\right)$, are listed in Table 2 . Variant chromosome 12 open reading frame 51 (C12orf51) rs11066280 showed the strongest association (beta $=-0.331, P=2.88 \times 10^{-6}$ in additive model). Out of these 12 SNPs, 4 SNPs were finally selected for the replication study using the Goldengate assay, through Tag SNP selection. The regional association plot of SNPs near C12orf51 and aldehyde dehydrogenase $2(A L D H 2)$ genes on chromosome 12q24 is shown in the Additional file 1: Figure S1. 
Table 1 General characteristics of the study subjects were included in final analysis

\begin{tabular}{|c|c|c|c|c|}
\hline & & $N(\%)$ & Dietary Pb ( $\mu \mathrm{g} /$ day) & Blood $\mathrm{Pb}^{\mathrm{a}}(\mu \mathrm{g} / \mathrm{dL})$ \\
\hline Total subjects & & 1,481 & $20.43 \pm 9.31$ & $2.21(2.17-2.26)$ \\
\hline \multirow[t]{3}{*}{ Gender } & Males & $640(43.2)$ & $21.89 \pm 10.10$ & $2.60(2.52-2.67)$ \\
\hline & Females & $841(56.8)$ & $19.31 \pm 8.51$ & $1.96(1.91-2.01)$ \\
\hline & & & $P<0.001$ & $P<0.001$ \\
\hline \multirow[t]{6}{*}{ Age groups } & -29 & $254(17.2)$ & $18.34 \pm 9.03$ & $1.66(1.59-1.74)$ \\
\hline & $30-39$ & $265(17.9)$ & $19.80 \pm 8.29$ & $2.05(1.97-2.14)$ \\
\hline & $40-49$ & $341(23.0)$ & $21.63 \pm 8.86$ & $2.15(2.07-2.24)$ \\
\hline & $50-59$ & $331(22.3)$ & $21.77 \pm 9.99$ & $2.56(2.47-2.66)$ \\
\hline & $60+$ & $290(19.6)$ & $19.88 \pm 9.76$ & $2.66(2.56-2.77)$ \\
\hline & & & $P<0.001$ & $P<0.001$ \\
\hline \multirow[t]{4}{*}{ Smoking history } & Non-smokers & $962(65.0)$ & $19.99 \pm 8.93$ & $2.03(1.98-2.08)$ \\
\hline & Ex-smokers & $243(16.4)$ & $21.90 \pm 9.54$ & $2.60(2.48-2.72)$ \\
\hline & Current-smokers & $276(18.6)$ & $20.67 \pm 10.28$ & $2.61(2.50-2.72)$ \\
\hline & & & $P=0.015$ & $P<0.001$ \\
\hline \multirow[t]{3}{*}{ Alcohol use } & Non-drinkers & $361(24.4)$ & $19.36 \pm 8.63$ & $2.07(1.99-2.14)$ \\
\hline & Drinkers & $1120(75.6)$ & $20.77 \pm 9.50$ & $2.26(2.21-2.32)$ \\
\hline & & & $P=0.009$ & $P<0.001$ \\
\hline
\end{tabular}

${ }^{a}$ Data presented as geometric mean with $95 \%$ confidence intervals

Table 3 shows dietary $\mathrm{Pb}$ intake and blood $\mathrm{Pb}$ levels according to the genotypes for the four SNPs analyzed via the replication study. Dietary $\mathrm{Pb}$ intake levels were not found to vary by SNP. However, we observed significant differences in blood $\mathrm{Pb}$ levels according to the three SNPs [C12orf51 rs11066280, myosin light chain 2 (MYL2) rs12229654, and ALDH2 rs671]. Moreover, the Spearman correlation coefficients between dietary $\mathrm{Pb}$ intake and blood $\mathrm{Pb}$ levels differed across the genotypes for these three SNPs.
After adjustment for potential confounders (e.g. age, sex and smoking status), three SNPs, namely C12orf51 rs11066280, MYL2 rs12229654, and ALDH2 rs671, associated with blood $\mathrm{Pb}$ level with suggestive significance. In the case of C12orf51 rs11066280, MYL2 rs12229654, and $A L D H 2$ rs671 polymorphisms, blood $\mathrm{Pb}$ levels were found to decrease significantly as the number of variant alleles increased (Table 4).

The C12orf51, MYL2, and ALDH2 SNPs were found to be highly related to alcohol consumption [21]; therefore,

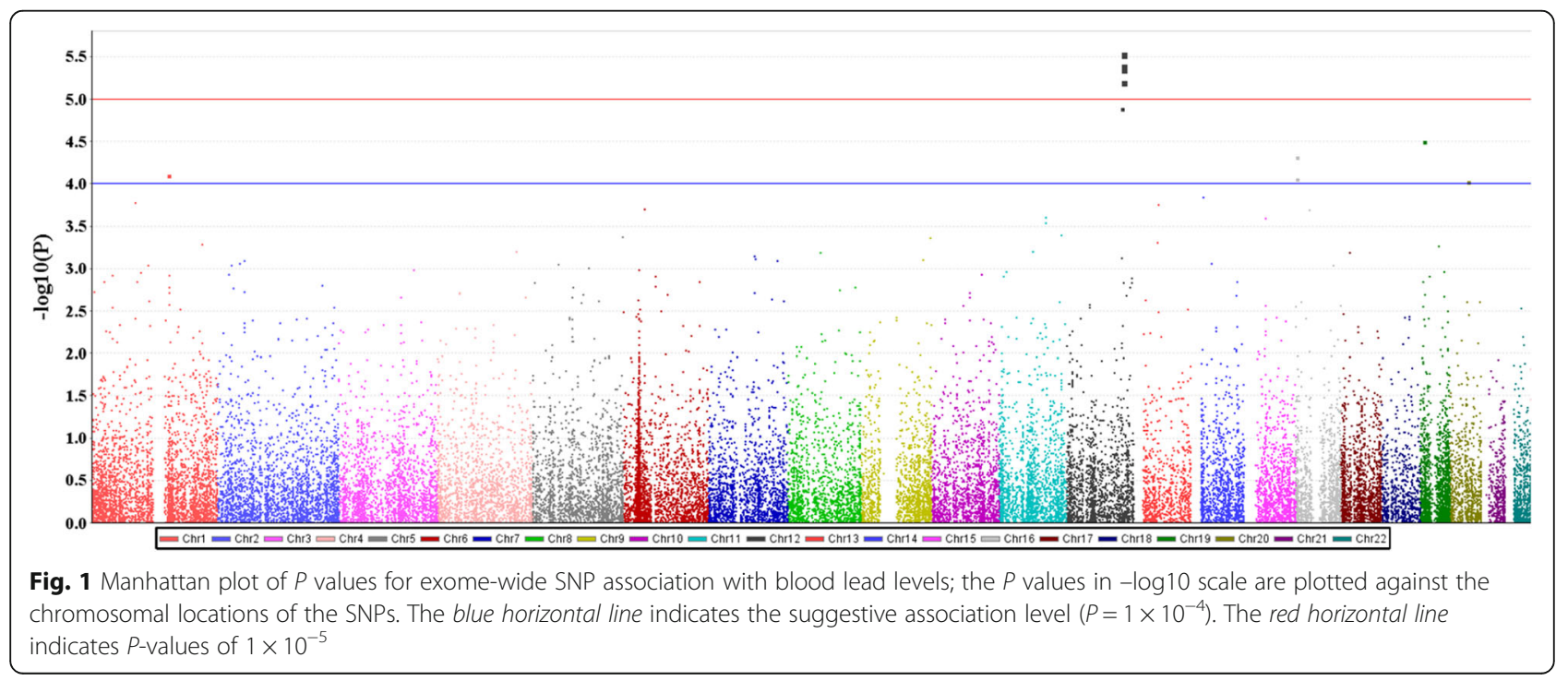


Table 2 The 12 single nucleotide polymorphisms associated with blood lead levels at a suggestive significance level $\left(P<1 \times 10^{-4}\right)$, as identified by exome-wide screening

\begin{tabular}{|c|c|c|c|c|c|c|c|c|c|}
\hline rs ID & Chr. & Position & Nearest gene & SNP type & Variant allele & MAF & $\beta^{a}$ & SE & $P$-value \\
\hline rs11066280 & 12 & 111302166 & C12orf51 & intronic & $A$ & 0.170 & -0.331 & 0.070 & $2.88 \times 10^{-6}$ \\
\hline rs2074356 & 12 & 111129784 & C12orf51 & intronic & $A$ & 0.140 & -0.348 & 0.074 & $2.98 \times 10^{-6}$ \\
\hline rs11066015 & 12 & 110652392 & ACAD10 & intronic & $A$ & 0.156 & -0.329 & 0.071 & $3.97 \times 10^{-6}$ \\
\hline rs671 & 12 & 110726149 & $A L D H 2$ & nonsynonymous & $A$ & 0.155 & -0.328 & 0.071 & $4.40 \times 10^{-6}$ \\
\hline rs3782886 & 12 & 110594872 & BRAP & synonymous & G & 0.165 & -0.319 & 0.070 & $6.27 \times 10^{-6}$ \\
\hline rs12229654 & 12 & 109898844 & MYL2 & intergenic & C & 0.139 & -0.331 & 0.075 & $1.28 \times 10^{-5}$ \\
\hline rs2228539 & 19 & 6877378 & EMR1 & nonsynonymous/splicing & G & 0.015 & 0.903 & 0.215 & $3.18 \times 10^{-5}$ \\
\hline rs2745099 & 16 & 1477459 & PTX4 & nonsynonymous & $A$ & 0.095 & -0.354 & 0.086 & $4.80 \times 10^{-5}$ \\
\hline rs41268474 & 1 & 150959136 & C1orf68 & nonsynonymous & $A$ & 0.058 & 0.441 & 0.111 & $7.90 \times 10^{-5}$ \\
\hline rs2667672 & 16 & 1476381 & PTX4 & nonsynonymous & A & 0.118 & -0.317 & 0.080 & $8.76 \times 10^{-5}$ \\
\hline rs2745097 & 16 & 1476500 & PTX4 & nonsynonymous & A & 0.118 & -0.317 & 0.080 & $8.76 \times 10^{-5}$ \\
\hline rs6126559 & 20 & 35990690 & VSTM2L & intronic & $A$ & 0.401 & 0.217 & 0.055 & $9.50 \times 10^{-5}$ \\
\hline
\end{tabular}

Chr chromosome, MAF minor allele frequency, SE standard error

${ }^{a}$ beta coefficient was adjusted for age, sex and smoking status by multiple linear regression analysis with an additive genetic model

we performed stratified analyses to assess the association between the SNPs and blood Pb levels according to alcohol drinking status. In non-drinkers, the chromosome 1 open reading frame 68 (C1orf68) rs41268474 was associated with blood Pb level. However, C12orf51 rs11066280, MYL2 rs12229654, and $A L D H 2$ rs671 polymorphisms were associated with blood $\mathrm{Pb}$ level only in drinkers. Although not statistically significant, a weak interaction was observed between alcohol drinking and the ALDH2 rs671 genotype with respect to blood $\mathrm{Pb}$ levels $(P$ for interaction $=0.067)$ (Table 5).

We additionally performed stratified analyses according to the dietary calcium intake, as calcium was related to both blood $\mathrm{Pb}$ level and alcohol drinking [22, 23]. Interestingly, in the high calcium-intake group, C12orf51 rs11066280, MYL2 rs12229654, and $A L D H 2$ rs671 polymorphisms were not associated with blood $\mathrm{Pb}$ levels. However, potential associations between these SNPs and

Table 3 Dietary lead intake, blood lead levels, and their correlation coefficient according to the four selected SNPs analyzed via the replication study

\begin{tabular}{|c|c|c|c|c|c|c|}
\hline SNP ID & Gene symbol & Genotype & $N$ & Dietary $\mathrm{Pb}$ & Blood Pb & Spearman's correlation coefficients \\
\hline \multirow[t]{4}{*}{ rs41268474 } & Clorf68 & GG & 1284 & $20.34 \pm 9.21$ & $2.21(1.48)$ & $0.097^{* *}$ \\
\hline & & GA & 193 & $20.51 \pm 9.33$ & $2.22(1.49)$ & 0.091 \\
\hline & & AA & 4 & $28.21 \pm 15.93$ & $2.12(1.59)$ & 0.712 \\
\hline & & & & $P=0.960$ & $P=0.958$ & \\
\hline \multirow[t]{4}{*}{ rs11066280 } & C12orf51 & $\pi$ & 1001 & $20.36 \pm 9.31$ & $2.25(1.49)$ & $0.103^{* *}$ \\
\hline & & TA & 433 & $20.38 \pm 8.98$ & $2.14(1.46)$ & $0.112^{*}$ \\
\hline & & AA & 47 & $20.80 \pm 10.49$ & $2.04(1.42)$ & -0.121 \\
\hline & & & & $P=0.951$ & $P=0.027$ & \\
\hline \multirow[t]{4}{*}{ rs12229654 } & MYL2 & $\pi$ & 1087 & $20.39 \pm 9.33$ & $2.25(1.49)$ & 0.127 \\
\hline & & TG & 353 & $20.31 \pm 8.86$ & $2.13(1.46)$ & 0.019 \\
\hline & & GG & 27 & $19.30 \pm 10.38$ & $1.92(1.37)$ & -0.220 \\
\hline & & & & $P=0.873$ & $P=0.015$ & \\
\hline \multirow[t]{4}{*}{ rs671 } & $\mathrm{ALDH} 2$ & GG & 1053 & $20.40 \pm 9.33$ & $2.26(1.49)$ & $0.120^{* *}$ \\
\hline & & GA & 388 & $20.24 \pm 8.86$ & $2.11(1.46)$ & 0.064 \\
\hline & & $\mathrm{AA}$ & 40 & $21.32 \pm 10.79$ & $1.98(1.38)$ & -0.210 \\
\hline & & & & $P=0.773$ & $P=0.003$ & \\
\hline
\end{tabular}


Table 4 Statistical significance of replicated SNPs for blood lead levels according to the univariate- or multivariate- regression models from replication analysis

\begin{tabular}{|c|c|c|c|c|c|c|c|c|c|c|}
\hline \multirow[t]{2}{*}{ SNP ID } & \multirow{2}{*}{$\begin{array}{l}\text { Gene } \\
\text { symbol }\end{array}$} & \multicolumn{3}{|c|}{ Univariate } & \multicolumn{3}{|c|}{ Multivariate model $1^{\mathrm{a}}$} & \multicolumn{3}{|c|}{ Multivariate model $2^{b}$} \\
\hline & & $\beta$ & SE & $P$-value & $\beta$ & SE & $P$-value & $\beta$ & SE & $P$-value \\
\hline rs41268474 & C1orf68 & 0.011 & 0.072 & 0.878 & 0.085 & 0.062 & 0.168 & 0.090 & 0.061 & 0.144 \\
\hline rs11066280 & C12orf51 & -0.134 & 0.046 & 0.004 & -0.170 & 0.040 & $1.94 \times 10^{-5}$ & -0.116 & 0.041 & 0.004 \\
\hline rs12229654 & MYL2 & -0.158 & 0.052 & 0.002 & -0.184 & 0.044 & $3.46 \times 10^{-5}$ & -0.130 & 0.045 & 0.004 \\
\hline rs671 & $\mathrm{ALDH} 2$ & -0.172 & 0.048 & $3.74 \times 10^{-4}$ & -0.197 & 0.041 & $1.73 \times 10^{-6}$ & -0.139 & 0.043 & 0.001 \\
\hline
\end{tabular}

SNPs were coded using an additive genetic model

${ }^{a}$ Adjusted for age, sex and smoking status

${ }^{\mathrm{b}}$ Adjusted for age, sex, smoking status, alcohol drinking and dietary lead intake

blood $\mathrm{Pb}$ level were observed in the low calcium-intake group (Table 6).

\section{Discussion}

In the present study, we identified three SNPs (i.e., C12orf51 rs11066280, MYL2 rs12229654, and ALDH2 rs671) on chromosome 12 that were associated with blood $\mathrm{Pb}$ levels in the Korean population. These three SNPs have been previously shown to be associated with alcohol consumption [21, 24], and are involved in the determination of $\mathrm{Pb}$ body burden in the Korean population via regulation of alcohol drinking behavior, especially in individuals with low calcium intake. To the best of our knowledge, this study presents the first evidence of a potential association between genetic factors and $\mathrm{Pb}$ body burden in the Asian population, using exome-wide association screening.

To date, several studies have performed genome-wide analyses to study the effects of genetic factors on individual differences in blood $\mathrm{Pb}$ concentration [17, 25, 26]. A previous genetic linkage analysis for 2,962 Australian adult twins reported that genetic variation with respect to the near solute carrier family 4 , sodium bicarbonate cotransporter, member 7 (SLC4A7) gene locus on chromosome 3 plays an important role in determining blood $\mathrm{Pb}$ concentration, after excluding shared environmental effects [25]. The first GWAS for cohorts from the UK and Australia identified the $A L A D$ rs 1805313 polymorphism on chromosome 9 affects blood $\mathrm{Pb}$ levels [17]. However, other GWAS have reported that no SNPs reached the threshold for statistical significance $\left(P<4.5 \times 10^{-9}\right)[26]$.
The finding of an association between SLC4A7 and blood $\mathrm{Pb}$ level was not replicated by other studies. On the other hand, some studies have reported that $A L A D$ genetic polymorphisms are associated with $\mathrm{Pb}$ body burden or toxicity, as the enzyme encoded by this gene, which is involved in the biosynthesis of heme, is inhibited by $\mathrm{Pb}$ $[15,16,27]$. In the present exome-wide screening analysis, we tested two SNPs (rs1805313 and rs1800435) of ALAD and 5 SNPs (rs2642926, rs3755652, rs4973768, rs75615379, and rs9854207) of SLC4A7; however, these SNPs did not achieve the suggestive level of association applied in this study $\left(P<1 \times 10^{-4}\right)$. This discrepancy may be attributed to the ethnic differences in terms of heavy metal levels [28] and genetic polymorphisms of heavy-metal-related genes [29]. A meta-analysis suggested that a significant association between $A L A D$ genetic polymorphisms and blood $\mathrm{Pb}$ level is limited only to the high- $\mathrm{Pb}$ exposure group [27]. These results suggest that the role of genetic factors in determining the body burden of $\mathrm{Pb}$ may vary according to the level of environmental exposure.

In the present study, we demonstrated a suggestively significant association between C12orf51 rs11066280, MYL2 rs12229654, and ALDH2 rs671 polymorphisms and blood $\mathrm{Pb}$ levels in 1,481 Korean subjects. Interestingly, all three SNPs were highly related to alcohol consumption [21, 24]. ALDH2 rs671, a non-synonymous SNP that occurs within a coding region, is located at position 487 (Glu > Lys), while C12orf51 rs11066280 and MYL2 rs12229654 are located in the intron and intergenic regions, respectively. Individuals with the $A L D H 2$ rs671 variant allele exhibit markedly decreased ALDH2

Table 5 Association between the replicated SNPs and blood lead levels according to alcohol consumption status

\begin{tabular}{|c|c|c|c|c|c|c|c|c|}
\hline \multirow[t]{2}{*}{ SNP ID } & \multirow{2}{*}{$\begin{array}{l}\text { Gene } \\
\text { symbol }\end{array}$} & \multicolumn{3}{|c|}{ Non-drinkers $(N=361)$} & \multicolumn{3}{|c|}{ Drinkers $(N=1120)$} & \multirow[b]{2}{*}{$P_{\text {for interaction }}$} \\
\hline & & $\overline{\beta^{a}}$ & SE & $P$-value & $\overline{\beta^{a}}$ & SE & $P$-value & \\
\hline rs41268474 & Clorf68 & 0.278 & 0.111 & 0.012 & 0.024 & 0.072 & 0.741 & 0.099 \\
\hline rs11066280 & C12orf51 & -0.068 & 0.062 & 0.275 & -0.137 & 0.053 & 0.010 & 0.191 \\
\hline rs12229654 & MYL2 & -0.073 & 0.068 & 0.284 & -0.157 & 0.058 & 0.007 & 0.369 \\
\hline rs671 & $A L D H 2$ & -0.069 & 0.062 & 0.266 & -0.182 & 0.057 & 0.002 & 0.067 \\
\hline
\end{tabular}

SNPs were coded using an additive genetic model

${ }^{a}$ Adjusted for age, sex, smoking status and dietary lead intake 
Table 6 Association between the replicated SNPs and blood lead levels according to dietary calcium intake

\begin{tabular}{|c|c|c|c|c|c|c|c|c|c|c|c|}
\hline \multirow[t]{3}{*}{ SNP ID } & \multirow{3}{*}{$\begin{array}{l}\text { Gene } \\
\text { symbol }\end{array}$} & \multicolumn{9}{|c|}{ Dietary calcium intake, mg/day } & \multirow[t]{3}{*}{$P_{\text {for interaction }}$} \\
\hline & & \multicolumn{3}{|c|}{ Low $(<473)(N=494)$} & \multicolumn{3}{|c|}{ Medium $(473-760)(N=493)$} & \multicolumn{3}{|c|}{ High $(>760)(N=494)$} & \\
\hline & & $\overline{\beta^{a}}$ & SE & $P$-value & $\overline{\beta^{a}}$ & SE & $P$-value & $\overline{\beta^{a}}$ & SE & $P$-value & \\
\hline rs41268474 & Clorf68 & 0.046 & 0.111 & 0.679 & 0.131 & 0.098 & 0.181 & 0.078 & 0.111 & 0.484 & 0.776 \\
\hline rs11066280 & C12orf51 & -0.147 & 0.073 & 0.043 & -0.126 & 0.072 & 0.081 & -0.066 & 0.069 & 0.335 & 0.661 \\
\hline rs12229654 & MYL2 & -0.154 & 0.077 & 0.047 & -0.141 & 0.082 & 0.084 & -0.089 & 0.078 & 0.253 & 0.821 \\
\hline rs671 & $\mathrm{ALDH} 2$ & -0.165 & 0.077 & 0.033 & -0.155 & 0.074 & 0.037 & -0.094 & 0.072 & 0.190 & 0.797 \\
\hline
\end{tabular}

SNPs were coded using an additive genetic model

${ }^{a}$ Adjusted for age, sex, smoking status, drink and dietary lead intake

enzyme activity and inhibited detoxification of toxic acetaldehyde, which is the first and most toxic metabolite of ethanol [24, 30]. Therefore, alcohol drinking behavior and dependency are associated with the ALDH2 rs671 SNP [31, 32]. Although C12orf51 rs11066280 and MYL2 rs12229654 were not located in a functional element, these two SNPs exhibit strong linkage disequilibrium with SNPs associated with alcohol drinking behavior [21, 33]. In our data, no differences in dietary $\mathrm{Pb}$ intake according to the genotypes of these alcohol-related genes were found; however, a difference was observed in the correlation of dietary $\mathrm{Pb}$ intake and blood $\mathrm{Pb}$ level according to these genotypes. Moreover, when stratified by drinking status, a potential association of C12orf51 rs11066280, $M Y L 2$ rs12229654, and $A L D H 2$ rs671 with blood $\mathrm{Pb}$ level was observed only in drinkers. A marginally significant gene-environment interaction between $A L D H 2$ rs671 and alcohol drinking on blood $\mathrm{Pb}$ level was found. Therefore, our data indicate that the association between the three SNPs investigated and blood Pb levels is mediated by alcohol drinking behavior.

Numerous studies have established links between higher blood $\mathrm{Pb}$ levels and alcohol consumption [13, 23, 34-36]. Consistent with their findings, the present data also found increased levels of blood $\mathrm{Pb}$ in alcohol drinkers $(2.26 \mu \mathrm{g} / \mathrm{dL})$ relative to non-drinkers $(2.07 \mu \mathrm{g} / \mathrm{dL})$. In addition, dietary $\mathrm{Pb}$ exposure level of drinkers (20.77 $\mu \mathrm{g} /$ day) was significantly higher than in non-drinkers (19.36 $\mu \mathrm{g} /$ day), although alcoholic beverages have low $\mathrm{Pb}$ concentrations $(11.13 \mu \mathrm{g} / \mathrm{kg})$ and contribute to only about $6.5 \%$ of total dietary $\mathrm{Pb}$ intake. These findings indicate that an increase in blood $\mathrm{Pb}$ levels in alcohol drinkers, rather than an increase in direct exposure to $\mathrm{Pb}$ via alcohol consumption, is associated with a variety of unhealthy lifestyle behaviors as a result of clustering tendency [37, 38]. Various unhealthy behaviors are considered to act as pivotal factors in increasing blood $\mathrm{Pb}$ concentrations.

In addition, it is considered that several possible biological mechanisms, such as the regulation of iron metabolism [39], decreased immunity [40, 41], and increased calcium excretion [23], underlie the effects of alcohol drinking on increased blood $\mathrm{Pb}$ levels. Firstly, alcohol consumption greatly increases $\mathrm{Pb}$ absorption by damaging the body's ability to regulate the absorption of iron. Further, the levels of hepcidin, which is involved in iron metabolism, are decreased by alcohol consumption. As iron and $\mathrm{Pb}$ compete for absorption, alcohol consumption may result in increased $\mathrm{Pb}$ absorption [39]. In addition, $\mathrm{Pb}$ is eliminated by macrophages, and it has recently been reported that macrophages and monocytes remove nanoparticles of specific heavy metals [42, 43]. Although it is well known that chronic $\mathrm{Pb}$ exposure decreases immune function [40, 41], our data suggest that a decrease in immunity, caused by a predisposing factor such as high alcohol intake, may increase the absorption of $\mathrm{Pb}$. Lastly, calcium is one of the most important nutritional factors in relation to the uptake of heavy metals, and calcium homeostasis is disrupted by alcohol consumption [44]; calcium deficiency resulting from alcohol consumption increases $\mathrm{Pb}$ absorption as the two ions are transported completely by the same transporter [45].

In this study, the effect of three alcohol-related SNPs on blood $\mathrm{Pb}$ levels was found to be dependent on the daily levels of calcium intake. Significant genetic effects on blood $\mathrm{Pb}$ levels were observed in the low calciumintake group but not in the high calcium-intake group. Our data were consistent with the findings of Pizent et al., who reported that alcohol consumption and low calcium intake are associated with increased blood $\mathrm{Pb}$ levels [23]. These data indicate that genetic susceptibility to high blood $\mathrm{Pb}$ levels may be mitigated if calcium depletion due to alcohol consumption were appropriately compensated by the intake of calcium-rich foods or supplements.

This study has strengths and limitations; the main strength was the use of a representative sample of the general Korean population with no history of occupational exposure to $\mathrm{Pb}$. Moreover, this study used the probable dietary intake levels of $\mathrm{Pb}$ to test the independent genetic effect. This study has several limitations, such as the limited statistical power of exome-wide screening because of the small sample size. To overcome this limitation, we used a two-stage approach, involving screening and replication; this increased power for the detection of genetic 
associations. In addition, the Bonferroni correction was applied for multiple tests, and a threshold for statistical significance was set. Nonetheless, none of SNPs showed an association with exome-wide significance $\left(P<1 \times 10^{-6}\right)$ in the screening stage. Four SNPs showed a potential association with blood $\mathrm{Pb}$ levels and achieved the level of suggestive association used in this study. Therefore, we could not exclude the possibility of false positives in this process. However, the same associations were confirmed in the 2-step test as well. Moreover, the biological plausibility of association between the suggested SNPs and the variation of blood $\mathrm{Pb}$ concentration in humans supports our results. Another limitation of this study is that we did not conduct the replication analysis in another independent cohort; therefore, the SNPs identified in the current study require replication in independent cohorts.

\section{Conclusions}

The present exome-wide association study demonstrates that C12orf51 rs11066280, MYL2 rs12229654, and ALDH2 rs671 polymorphisms are linked to blood $\mathrm{Pb}$ levels in the Korean population. These three SNPs are involved in the determination of the $\mathrm{Pb}$ body burden in Koreans via regulation of alcohol drinking behavior, and their negative effects may be compensated by appropriate calcium intake. Our results suggest that individuals with genetic susceptibility to elevated $\mathrm{Pb}$ body burden must take measures to avoid environmental exposure to $\mathrm{Pb}$ and maintain a healthy lifestyle, that is, avoid smoking and drinking and appropriate calcium intake.

\section{Additional file}

Additional file 1: Results of exome-wide association study for screening of genetic variability for blood lead levels; Table S1. Top 100 single nucleotide polymorphisms most significantly associated with blood lead levels, as identified by the exome-wide association study; Figure S1. Regional association plot of single nucleotide polymorphisms near C12orf51 and ALDH2 genes on chromosome 12q24. (DOCX $139 \mathrm{~kb}$ )

\section{Abbreviations}

ALAD: Aminolevulinate dehydratase; ALDH2: Aldehyde dehydrogenase 2; C12orf51: Chromosome 12 open reading frame 51; GM: Geometric mean; GSD: Geometric standard deviation; GWAS: Genome-wide association study; HWE: Hardy-Weinberg equilibrium; MAF: Minor allele frequency; MYL2: Myosin light chain 2; SLC4A7: Solute carrier family 4, sodium bicarbonate cotransporter, member 7; SNP: Single nucleotide polymorphism; VDR: Vitamin D receptor

\section{Acknowledgements}

Not applicable.

\section{Funding}

This research was supported by Grant No.13162MFDS778 from the Ministry of Food and Drug Safety in 2014.

\section{Availability of data and materials}

The datasets supporting the conclusions of this article are included within the article.

\section{Authors' contributions}

SYE and JAL conducted the statistical analyses. MSH, HJK, BSC, JDP, HK, and YDK conceived and designed the study, and interpreted the data. SYE and YDK wrote the draft and revised the manuscript. All authors read and approved the final manuscript.

\section{Competing interests}

The authors declare that they have no competing interests.

\section{Consent for publication}

Not applicable.

\section{Ethics approval and consent to participate}

This study was approved by the Institutional Review Board of Dankook University Hospital, Republic of Korea (IRB No. 2013-03-008), and informed consent was obtained from all individual participants.

\section{Author details}

'Department of Preventive Medicine, College of Medicine, Chungbuk National University, 1 Chungdae-Ro, Seowon-Gu, Cheongju, Chungbuk 28644, Korea. ${ }^{2}$ Food Risk Analysis Division, National Institute of Food and Drug Safety Evaluation, 187 Osongsaengmyeong 2-Ro, Heungdeok-Gu, Cheongju 28159, Korea. ${ }^{3}$ Department of Preventive Medicine, Dankook University College of Medicine, 119 Dandae-Ro, Dongnam-Gu, Cheonan, Chungnam 31116, Korea. ${ }^{4}$ Department of Preventive Medicine, Chung-Ang University College of Medicine, 84 Heukseok-Ro, Dongjak-Gu, Seoul 06974, Korea.

Received: 6 August 2016 Accepted: 14 February 2017

Published online: 17 February 2017

\section{References}

1. Were FH, Moturi MC, Gottesfeld P, Wafula GA, Kamau GN, Shiundu PM. Lead exposure and blood pressure among workers in diverse industrial plants in Kenya. J Occup Environ Hyg. 2014;11:706-15.

2. Ozturk MT, Yavuz B, Ozkan S, Ayturk M, Akkan T, Ozkan E, et al. Lead exposure is related to impairment of aortic elasticity parameters. J Clin Hypertens (Greenwich). 2014;16:790-3.

3. Kim MG, Ryoo JH, Chang SJ, Kim CB, Park JK, Koh SB, et al. Blood Lead Levels and Cause-Specific Mortality of Inorganic Lead-Exposed Workers in South Korea. PLoS One. 2015;10:e0140360.

4. Apostoli P, Kiss P, Porru S, Bonde JP, Vanhoorne M. Male reproductive toxicity of lead in animals and humans. ASCLEPIOS Study Group. Occup Environ Med. 1998:55:364-74.

5. ul Haq N, Tabassum S, Anjum R, Fatima B. Lead, hypertension, and chronic renal failure. J Ayub Med Coll Abbottabad. 2013;25:96-9.

6. Kasten-Jolly J, Lawrence DA. Lead modulation of macrophages causes multiorgan detrimental health effects. J Biochem Mol Toxicol. 2014;28: 355-72.

7. Patrick L. Lead toxicity, a review of the literature. Part 1: Exposure, evaluation, and treatment. Altern Med Rev. 2006;11:2-22.

8. Bernhard D, Rossmann A, Wick G. Metals in cigarette smoke. IUBMB Life. 2005;57:805-9.

9. Jarup L. Hazards of heavy metal contamination. Br Med Bull. 2003;68:167-82

10. Panel EC. Cadmium in food - Scientific opinion of the Panel on Contaminants in the Food Chain., vol. 980. 2009.

11. Faroon O, Ashizawa A, Wright S, Tucker P, Jenkins K, Ingerman L, et al Agency for Toxic Substances and Disease Registry (ATSDR) Toxicological Profiles. In: Toxicological Profile for Cadmium. Atlanta: Agency for Toxic Substances and Disease Registry (US); 2012.

12. Rhie J, Lee HE. Physical Activity and Blood Lead Concentration in Korea: Study Using the Korea National Health and Nutrition Examination Survey (2008-2013). J Korean Med Sci. 2016;31:852-8.

13. Weyermann $\mathrm{M}$, Brenner $\mathrm{H}$. Alcohol consumption and smoking habits as determinants of blood lead levels in a national population sample from Germany. Arch Environ Health. 1997;52:233-9.

14. Lopez CM, Vallejo NE, Pineiro AE, Uicich R, Damin CF, Sarchi Ml, et al. Alteration of biochemical parameters related with exposure to lead in heavy alcohol drinkers. Pharmacol Res. 2002;45:47-50.

15. Onalaja AO, Claudio L. Genetic susceptibility to lead poisoning. Environ Health Perspect. 2000;108 Suppl 1:23-8. 
16. da Cunha Martins Jr A, Mazzaron Barcelos GR, Jacob Ferreira AL, de Souza MF, de Syllos Colus IM, Antunes LM, et al. Effects of Lead Exposure and Genetic Polymorphisms on ALAD and GPx Activities in Brazilian Battery Workers. J Toxicol Environ Health A. 2015;78:1073-81.

17. Warrington NM, Zhu G, Dy V, Heath AC, Madden PA, Hemani G, et al. Genome-wide association study of blood lead shows multiple associations near ALAD. Hum Mol Genet. 2015;24:3871-9.

18. Jhun MA, Hu H, Schwartz J, Weisskopf MG, Nie LH, Sparrow D, et al. Effect modification by vitamin $D$ receptor genetic polymorphisms in the association between cumulative lead exposure and pulse pressure: a longitudinal study. Environ Health. 2015;14:5.

19. Eom SY, Choi SH, Ahn SJ, Kim DK, Kim DW, Lim JA, et al. Reference levels of blood mercury and association with metabolic syndrome in Korean adults. Int Arch Occup Environ Health. 2014;87:501-13.

20. Lim JA, Kwon HJ, Ha M, Kim H, Oh SY, Kim JS, et al. Korean research project on the integrated exposure assessment of hazardous substances for food safety. Environ Health Toxicol. 2015;30:e2015004.

21. Baik I, Cho NH, Kim SH, Han BG, Shin C. Genome-wide association studies identify genetic loci related to alcohol consumption in Korean men. Am J Clin Nutr. 2011;93:809-16.

22. Suarez-Ortegon MF, Mosquera M, Caicedo DM, De Plata CA, Mendez F. Nutrients intake as determinants of blood lead and cadmium levels in Colombian pregnant women. Am J Hum Biol. 2013;25:344-50.

23. Pizent A, Jurasovie J, Telisman S. Blood pressure in relation to dietary calcium intake, alcohol consumption, blood lead, and blood cadmium in female nonsmokers. J Trace Elem Med Biol. 2001;15:123-30.

24. Wang RS, Nakajima T, Kawamoto T, Honma T. Effects of aldehyde dehydrogenase-2 genetic polymorphisms on metabolism of structurally different aldehydes in human liver. Drug Metab Dispos. 2002;30:69-73.

25. Whitfield JB, Dy V, McQuilty R, Zhu G, Montgomery GW, Ferreira MA, et al. Evidence of genetic effects on blood lead concentration. Environ Health Perspect. 2007;115:1224-30.

26. Ng E, Lind PM, Lindgren C, Ingelsson E, Mahajan A, Morris A, et al. Genomewide association study of toxic metals and trace elements reveals novel associations. Hum Mol Genet. 2015;24:4739-45.

27. Scinicariello F, Murray HE, Moffett DB, Abadin HG, Sexton MJ, Fowler BA Lead and delta-aminolevulinic acid dehydratase polymorphism: where does it lead? A meta-analysis. Environ Health Perspect. 2007;115:35-41.

28. McKelvey W, Gwynn RC, Jeffery N, Kass D, Thorpe LE, Garg RK, et al. A biomonitoring study of lead, cadmium, and mercury in the blood of New York city adults. Environ Health Perspect. 2007;115:1435-41.

29. Eom SY, Lim JA, Kim YD, Choi BS, Hwang MS, Park JD, et al. Allele frequencies of the single nucleotide polymorphisms related to the body burden of heavy metals in the Korean population and their ethnic differences. Toxicol Res. 2016;32:195-205.

30. Yokoyama T, Saito K, Lwin H, Yoshiike N, Yamamoto A, Matsushita Y, et al. Epidemiological evidence that acetaldehyde plays a significant role in the development of decreased serum folate concentration and elevated mean corpuscular volume in alcohol drinkers. Alcohol

Clin Exp Res. 2005;29:622-30.

31. Takeshita T, Morimoto K. Self-reported alcohol-associated symptoms and drinking behavior in three ALDH2 genotypes among Japanese university students. Alcohol Clin Exp Res. 1999;23:1065-9.

32. Kim DJ, Choi IG, Park BL, Lee BC, Ham BJ, Yoon S, et al. Major genetic components underlying alcoholism in Korean population. Hum Mol Genet. 2008; 17:854-8

33. Kumar V, Westra HJ, Karjalainen J, Zhernakova DV, Esko T, Hrdlickova B, et al. Human disease-associated genetic variation impacts large intergenic noncoding RNA expression. PLoS Genet. 2013;9:e1003201.

34. Shaper A, Pocock S, Walker M, Wale C, Clayton B, Delves H, et al. Effects of alcohol and smoking on blood lead in middle-aged British men. Br Med J (Clin Res Ed). 1982;284:299-302.

35. Hense HW, Filipiak B, Keil U. Alcohol consumption as a modifier of the relation between blood lead and blood pressure. Epidemiology. 1994;5: 120-3.

36. Schuhmacher M, Domingo JL, Llobet JM, Corbella J. Variability of blood lead levels in an urban population in relation to drinking and smoking habits. Sci Total Environ. 1993;138:23-9.

37. Noble N, Paul C, Turon H, Oldmeadow C. Which modifiable health risk behaviours are related? A systematic review of the clustering of Smoking,
Nutrition, Alcohol and Physical activity ('SNAP') health risk factors. Prev Med. 2015;81:16-41.

38. Ma J, Betts NM, Hampl JS. Clustering of lifestyle behaviors: the relationship between cigarette smoking, alcohol consumption, and dietary intake. Am J Health Promot. 2000;15:107-17.

39. Mena NP, Esparza A, Tapia V, Valdes P, Nunez MT. Hepcidin inhibits apical iron uptake in intestinal cells. Am J Physiol Gastrointest Liver Physiol. 2008; 294:G192-8.

40. Mishra KP, Rani R, Yadav VS, Naik S. Effect of lead exposure on lymphocyte subsets and activation markers. Immunopharmacol Immunotoxicol. 2010;32: 446-9.

41. Vallverdu-Coll N, Ortiz-Santaliestra ME, Mougeot F, Vidal D, Mateo R. Sublethal $\mathrm{Pb}$ exposure produces season-dependent effects on immune response, oxidative balance and investment in carotenoid-based coloration in red-legged partridges. Environ Sci Technol. 2015;49:3839-50.

42. Bartneck M, Keul HA, Zwadlo-Klarwasser G, Groll J. Phagocytosis independent extracellular nanoparticle clearance by human immune cells. Nano Lett. 2010;10:59-63.

43. Bartneck M, Keul HA, Singh S, Czaja K, Bornemann J, Bockstaller M, et al. Rapid uptake of gold nanorods by primary human blood phagocytes and immunomodulatory effects of surface chemistry. ACS Nano. 2010;4:3073-86.

44. Hirsch P, Peng TC. Calcium and Phosphorus in Health and Disease. Boca Raton: CRC Press; 1996.

45. Simons TJ. Lead-calcium interactions in cellular lead toxicity. Neurotoxicology. 1993;14:77-85.

\section{Submit your next manuscript to BioMed Central and we will help you at every step:}

- We accept pre-submission inquiries

- Our selector tool helps you to find the most relevant journal

- We provide round the clock customer support

- Convenient online submission

- Thorough peer review

- Inclusion in PubMed and all major indexing services

- Maximum visibility for your research

Submit your manuscript at www.biomedcentral.com/submit
Biomed Central 